\title{
Tecnologías de la Información y la Comunicación (TIC) para fortalecer el aprendizaje significativo de los estudiantes
}

\author{
Importance of incorporating Information and Communication Technologies (ICT) to strengthen \\ the significant learning of students
}

María L. Sánchez-Padilla ${ }^{a}$, Margarita Lazcano Ortiz ${ }^{b}$,

José Arias-Rico ${ }^{c}$, Reyna C. Jiménez-Sánchez ${ }^{b}$

\begin{abstract}
:
The vertiginous advance of science and information and communication technologies (ICT), enrich learning opportunities, it is vital to develop a vision for the future because traditional methods are less and less effective and for many Universities remain the main space for acquiring knowledge, values, socialization mechanisms as well as computers and the internet, therefore global universities must privilege synchronous and asynchronous spaces, promoting public policy efforts to achieve significant, relevant and quality learning mediated by technologies to respond to the needs of a globalized world.
\end{abstract}

Keywords:

Synchronous, asynchronous, meaningful learning

\section{Resumen:}

El avance vertiginoso de la ciencia y las tecnologías de la información y la comunicación (TIC), enriquecen las oportunidades de aprendizaje, siendo de vital importancia desarrollar una visión de futuro porque los métodos tradicionales son cada vez menos efectivos y para muchos las Universidades siguen siendo el principal espacio para adquirir conocimientos, valores, mecanismos de socialización así como también computadoras e internet, por lo consiguiente las Universidades globales deben privilegiar espacios sincrónicos y asincrónicos, potenciando esfuerzos de política pública para lograr aprendizajes significativos, pertinentes y de calidad mediados por tecnologías para responder a las necesidades de un mundo globalizado.

\section{Palabras Clave:}

Sincrónico, asincrónico, aprendizaje significativo

\section{Introducción}

Los contextos universitarios son espacios de reflexión e innovación para garantizar las competencias específicas y genéricas que permea el plan de estudios, para lograr la pertinencia de los programas educativos. Entre los recursos es de vital importancia la incorporación de las (TIC), como palanca de cambio profundo, para atender las diferentes culturas, con marcos flexibles, que se adapten a las necesidades, con trabajo colaborativo, conformando redes con distintos niveles de complejidad, en los que se revalué la presencia física, imaginar otros escenarios aula invertida, que permita cambiar la cultura memorística, que no solo se busque la información, sino que se procese, se transfiera que les permita a mediadores y estudiantes la estrategia de solucionar problemas favoreciendo así la empleabilidad,

\footnotetext{
Autor de correspondencia, Universidad Autónoma del Estado de Hidalgo, Instituto de Ciencias de la Salud, Email: marialuisasanchezpadilla@yahoo.com

${ }^{\mathrm{b}}$ Universidad Autónoma del Estado de Hidalgo, Instituto de Ciencias de la Salud, Email: magos1000@ hotmail.com

${ }^{c}$ Universidad Autónoma del Estado de Hidalgo, Instituto de Ciencias de la Salud ,Email: josearias.rico@ @otmail.com

d Universidad Autónoma del Estado de Hidalgo, Instituto de Ciencias de la Salud, Email: cristyji@ hotmail.com
} 
al dar respuesta a las líneas estratégicas de los objetivos de desarrollo sostenible (ODS), inclusión, equidad, y calidad de los conocimientos para promover aprendizajes para toda la vida a través de reflexionar los nuevos paradigmas educativos desde donde se gestan las políticas públicas, investigadores, docentes, hacia enfoques centrados en el estudiante. (Aguerrondo \& Vaillant, 2015)

Las tecnologías son un paradigma innovador, ante los cambios vertiginosos en el desarrollo de las TIC, permite afirmar que las sociedades más avanzadas han entrado a la sociedad del conocimiento emergentes que se dan en un círculo virtuoso, en el cual los progresos del conocimiento producen a largo plazo más conocimiento, gracias a las innovaciones tecnológicas, los valores y prácticas de creatividad e innovación desempeñan un papel importante y fundamental para responder a las necesidades de la sociedad. (UNESCO, 2005)

Las Tecnologías de la Información y la comunicación (TIC), son poco utilizados por los docentes, con más o menores competencias digitales, pero a medida que aparece una amplia gama de fuentes y se consigue un fácil acceso a la información, se desplaza la noción de saber a la de saberes, la noción de verdad a la de verdades, distanciándose la concepción de saber monolítico o el saber constituido, para surgir una visión más cercana al "conocimiento en construcción" la cual no se ha logrado porque los docentes deben afrontar el cómo se enseña y el cómo se aprende, porque las tecnologías introducen nuevos elementos al discurso pedagógico desconocido inclusive por aquellos que ya se encuentran en procesos de interacción formativa en sistemas y redes digitales. (Duarte, 2003).Las tecnologías fortalecen la estrategia del autoaprendizaje porque permite a los estudiantes técnicas como el estudio individual, tareas individuales e investigación, permite un aprendizaje interactivo al escuchar conferencias, participan en los foros de discusión de los temas de interés y chatear, se logra un aprendizaje colaborativo que permite la resolución de problemas, participar en wikis, blogs entre otros, el estudiante al usar las tecnologías desarrolla actitudes de autonomía, autodirección y autorregulación en su proceso de aprender (UNED, 2005 )

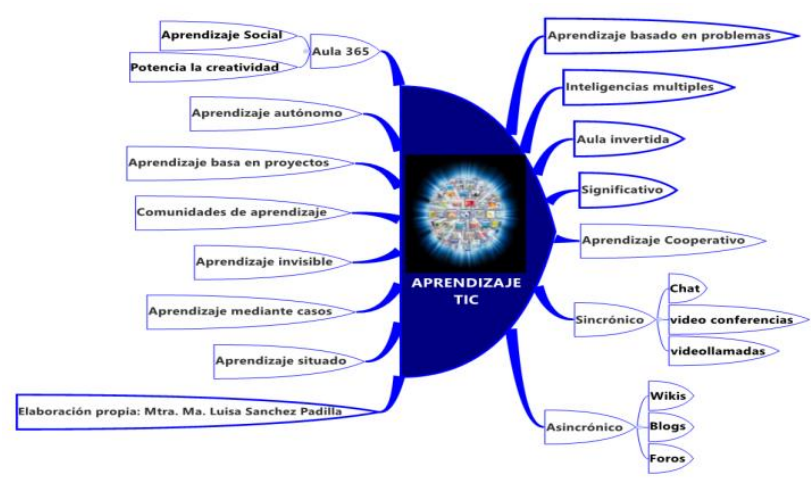

\section{CONCLUSIÓN}

En los últimos años, en el campo educativo, se habla de "nuevas tecnologías" a "tecnologías de la información y comunicación" y posteriormente a tecnologías para el aprendizaje y la comunicación. Estos cambios de lenguaje no son de carácter ornamental, sino que obedecen a un modo de entender los artefactos y dispositivos tecnológicos: y de enfatizar en estrategias diversas como el aprendizaje situado, colaborativo, basado en casos, y problemas, autónomo, significativo, aula invertida así como sincrónico y asincrónico ya no deben considerarse nuevos sino cotidianos porque el uso de la tecnología per se no es necesariamente buena sino se halla al servicio de un mayor aprendizaje y si no se combina con la experiencia, conocimiento y habilidad para el logro de mejores resultados académicos. 
Publicación semestral, Educación y Salud Boletín Científico Instituto de Ciencias de la Salud Universidad Autónoma del Estado de

Hidalgo, Vol. 8, No. 15 (2019) 116-118

\section{Referencias}

[1] Delors, J. (1996). La educación encierra un tesoro. Mexico D.F: Ediciones UNESCO.

[2] López Carrasco, M. Á. (2015). Aprendizaje Competencias y TIC, 3a edición. Mexico Distrito Federal : .Pearson Educación

[3] UNED (2005). El modelo pedagógico. Tomado desde http://estatico.uned.ac.cr/paa/pdf/Materiales-autoev/24.pdf

[4] UNESCO. (1987). Informe de la Comision Internacional para el Desarrollo de la Educación. Madrid: Alianza Editorial.

[5] UNESCO (2005). Hacia las sociedades del conocimiento. Informe mundial. http://www. flacso.edu.mx/colaboratorio/pdf/colaboratorio_unesco.pdf, recuperado 27/08/16.

[6] UNESCO. (2016). Resumen del informe de seguimiento de la educación en el mundo. La Educación al servicio de los pueblos y el planeta, Creación de futuros sostenibles para todos . Paris Francia: 